\title{
A utilização do software Tracker no ensino de física: um breve relato
}

\author{
Pereira, R. D' ${ }^{1}$ Santos, J.F. ${ }^{2}$

\begin{abstract}
1 Programa de Pós-Graduação em Ensino de Física (PPGEnFis), Universidade Federal do Espírito Santo, Vitória, ES, Brasil. Mateus, ES, Brasil.
\end{abstract} \\ 2 Mestranda do Programa de Pós-graduação em Ensino na Educação Básica (PPGEEB), Universidade Federal do Espírito Santo, São
}

* e-mail: rodrigo.pereira@ufes.br

\section{Resumo}

Diversas pesquisas têm sido realizadas com o objetivo de propor novas metodologias que possam auxiliar uma melhora da compreensão dos conteúdos de física. Neste contexto, a utilização de laboratórios experimentais e outros recursos didáticos como por exemplo vídeos e simuladores se tornam uma ferramenta fundamental. Neste trabalho usamos o software Tracker em uma aula de física objetivando dinamizar e maximizar o processo de ensino aprendizagem e tornar a aula mais dinâmica e mais próxima à realidade do aluno. Com a utilização deste software, foi ministrada uma aula para duas turmas do $1^{\circ}$ ano do ensino médio, para tal fizemos vídeos que retratavam alguns movimentos do cotidiano dos alunos. Para nortear o planejamento da aula, foi aplicado um teste diagnóstico e através dos resultados verificamos que os alunos possuem dificuldades em relacionar os conceitos físicos abordados com seus respectivos gráficos. Na sequência a aula foi planejada e executada. Após a aula foi aplicado um novo teste diagnóstico para verificar de forma qualitativa e quantitativa a utilização do software. Os resultados indicaram que o software Tracker é uma ferramenta muito útil para o ensino do comportamento gráfico das variáveis estudadas, pois, facilitou o entendimento dos conceitos abordados.

Palavras chave: Tracker, Gráficos, Ensino de Física

\section{Abstract}

Several researches have been carried out with the objective of proposing new methodologies that can help an improvement of the understanding of the concepts of physics. In this context, the use of experimental labs and other didactic resources such as videos and simulators become a fundamental tool. In this work we use Tracker software in a physics class with the purpose of dynamizing and maximizing the process of teaching learning and making the class more dynamic and closer to the student reality. With the use of this software, a class was taught for two classes of the 1st year of high school, for that we made videos that portrayed some movements of the daily life of the students. To guide the planning of the lesson, a diagnostic test was applied and through the results we verified that the students have difficulties in relating the physical concepts addressed with their respective graphs. in the sequence the lesson was planned and executed. In the aftermath of the class, a new diagnostic test was applied to verify the qualitative and quantitative use of the software. The reached results indicated that the Tracker software is a very useful tool to teach the graphic behavior of the studied variables, since it facilitated the understanding of the concepts discussed.

Keywords: Tracker, Graphs, Teaching physics.

\section{Introdução}

Atualmente na maioria das escolas o ensino da física quase sempre tem como principal objetivo a memorização de fórmulas e soluções algébricas de exercícios, tal fato já tinha sido observado por Barbosa em 2008 [1]. Isso ocorre devido ao fato de muitas vezes, a física ensinada nos anos finais da educação objetivar apenas preparar o aluno para a resolução de exercícios dos diversos processos seletivos utilizados hoje em dia.

Além disso, esta prática também é, possivelmente, uma das principais causas do distanciamento do aluno dessa disciplina, gerando dificuldades de aprendizagem, fazendo com que a mesma seja pouco entendida ou não muito 
apreciada [2]. Outro fator que contribui para a "marginalização" da disciplina de física é o fato de que muitas vezes as metodologias utilizadas para o ensino de Física serem pouco variadas, utilizando tradicionalmente quadro, giz e livro didático [3].

Quando o aluno deixa de aprender e apenas memoriza expressões matemáticas, ele encontra muitas dificuldades para associar o que se aprende em sala de aula com os fenômenos observados no seu diaa-dia, isto também ocasiona um grande desinteresse do aluno com relação ao ensino tradicional de física.

Atualmente, com o avanço tecnológico e suas ferramentas, como por exemplo, a internet, com um simples clique faz o impossível tornar-se palpável, como navegar pelo corpo humano e visualizar a Terra do espaço sem sair do lugar. Prender a atenção do aluno que está diretamente em contato com esse universo é muito complicado com aulas onde são utilizados apenas o quadro e o giz.

Diante da necessidade de mudança da metodologia de ensino da física objetivando uma aprendizagem significativa, a utilização de laboratórios experimentais e outros recursos didáticos como vídeos, simuladores entre outros recursos tecnológicos, que nos dias atuais estão acessíveis a maior parte dos estudantes e escolas, se torna uma ferramenta fundamental.

Considerando a grande variedade de recursos tecnológicos de baixo custo, uma proposta interessante é o uso de filmagem de câmeras digitais e celulares para registrar o movimento de objetos em situações de rotina, como por exemplo, os movimentos presentes em atividades esportivas e de lazer. Posteriormente com a ajuda de um software, essas imagens são transferidas para um computador, onde são analisadas e com isso é possível investigar o comportamento de algumas grandezas físicas e em alguns casos até mesmo propor uma modelagem física do problema.

Neste cenário, alguns destes recursos tecnológicos podem ser obtidos de forma gratuita na internet, entre eles o Tracker [4], que é um programa gratuito que analisa imagens e vídeos sobre movimentos e que fornece informações a respeito das grandezas físicas de interesse. Ressaltamos também, que a observação de imagens, tabelas e gráficos obtidos neste software fornece ao aluno elementos para verificação, e compreensão do comportamento das variáveis físicas do problema. Entretanto, é necessário que as análises feitas com o auxílio deste software promovam uma interação entre os conceitos estudados e o cotidiano do aluno. $\mathrm{Na}$ literatura encontramos diversos trabalhos que obtiveram êxito na promoção desta integração [5]. Convém ressaltar que o laboratório de física é de suma importância no ensino da Física, pois as simulações, as animações em computadores, na sua maioria não conseguem trazer a riqueza de detalhes e as dificuldades técnicas que somente no laboratório hão de ser vivenciadas [5].

Diante deste cenário, temos como principal objetivo relatar a utilização do software Tracker como um recurso auxiliar no ensino dos conceitos iniciais de cinemática e, além disso, fornecer indícios sobre o efeito da utilização deste tipo de ferramenta no processo de ensino-aprendizagem e na motivação dos alunos.

\section{O uso da tecnologia em sala de aula}

O uso da tecnologia na sala de aula está descrito nos parâmetros curriculares, como forma de desenvolver habilidade e competência nos alunos. Aliado a este fato, de acordo com Vygotsky, é na atividade prática, ou seja, na coletividade que a pessoa se aproveita da linguagem e dos objetos físicos disponíveis em sua cultura, promovendo assim seu desenvolvimento, dando ênfase aos conhecimentos histórico-cultural, produzidos e/ou já existentes em seu cotidiano.

Atualmente temos observado um grande avanço no desenvolvimento de softwares/programas que ao invés de realizar simulações fazem análises em tempo real.

Diversos estudos foram realizados com oobjetivo de verificar a eficiência do uso da análise de vídeos como recurso didático [6-8]. Em todos estes trabalhos, observou-se que 0 recurso era extremamente eficaz, e como resultado conseguiram que os estudantes se mostrassem muito mais motivados, gerando mais discussões na sala de aula e 


\section{Encontro Científico de Física Aplicada}

diminuindo as dúvidas sobre os conceitos fundamentais.

No ano de 2008, Calloni utilizou o software, o Tracker, em uma proposta que visava uma abordagem atrativa e motivadora de determinado conteúdo com os 135 alunos do $8^{\circ}$ ano de uma escola de rede privada, situada na cidade de Caxias do Sul. Com relação ao conteúdo de física a ser trabalhado o professor optou pela cinemática, com ênfase em análises gráficas e numéricas do comportamento das grandezas físicas: distância percorrida, aceleração, trajetória dentre outras.

Desta forma, observamos a importância do desenvolvimento de pesquisas a respeito de ferramentas que possam auxiliar o professor no sentido de reduzir a distância entre a teoria e o cotidiano do aluno.

\section{Desenvolvimento do trabalho}

No primeiro momento era necessário definirmos os conteúdos a serem abordados. Neste sentido, sabemos que as deficiências conceituais em física tem sido alvo de diversos trabalhos [6,8]. Os resultados destes trabalhos indicam uma grande deficiência conceitual nos conteúdos inicias de mecânica, portanto, optamos por abordar, neste trabalho, os conteúdos de velocidade e aceleração. Outro aspecto que consideramos é que os gráficos são uma poderosa ferramenta para o entendimento de conceitos de cinemática, contudo esta ferramenta não é dominada de forma apropriada pelos estudantes. Estes geralmente confundem o gráfico de posição em função do tempo com trajetória, ou não conseguem fazer a correta distinção entre os gráficos da posição, velocidade e aceleração em função do tempo, além disso, não demonstram compreensão do significado da área e do coeficiente angular dos respectivos gráficos $[6,8]$. Desta forma, utilizaremos o software Tracker na abordagem dos conteúdos: velocidade e aceleração associados ao comportamento gráfico destas grandezas, é importante, ressaltar que este programa já foi utilizado com sucesso em outros trabalhos $[5,8]$.

A fim de detectarmos especificamente as dificuldades conceituais dos alunos que iriam participar desta prática a respeito dos conceitos de velocidade $\mathrm{e}$ aceleração, decidimos aplicar um teste diagnostico composto de 5 questões retiradas do Mechanical Test Baseline [9]

Durante uma aula de física aplicamos o teste em 2 turmas do $1^{\circ}$ ano do ensino médio, totalizando 52 alunos participantes. O tempo disponibilizado para os alunos responderem as questões foi de 50 minutos e não foi permitida a utilização de calculadoras ou consulta a qualquer outro material, cabe pontuar que os todos alunos terminaram o teste dentro do tempo estipulado.

Com base nos resultados obtidos no teste diagnóstico, iniciamos a produção dos vídeos que seriam utilizados em sala de aula. Além disso, no processo de produção, optamos pela construção de vídeos que mostrassem situções do cotidiano dos alunos, de modo a permitir uma vivência ativa do processo de ensino-aprendizagem.

Desta forma, filmamos as seguintes situações: (i) uma pessoa andando devagar e depois depressa, (ii) movimento de uma pessoa correndo, (iii) movimento de uma pessoa no skate, (iv) movimento de uma pessoa andando de bicicleta na ida e volta. É importante destacar que todos os vídeos foram filmados em uma praça da cidade, e que também utilizamos vídeos, onde são mostrados motos em movimento, disponíveis na internet. Além disso, aplicamos na semana seguinte outro teste diagnóstico, nos mesmos moldes do $1^{\circ} \mathrm{e}$ abordando os mesmos conteúdos.

Na Figura 1, é mostrado a tela do software Tracker após a análise do movimento de um ciclista, na parte da esquerda temos o vídeo e na parte da direita temos a análise gráfica do movimento.

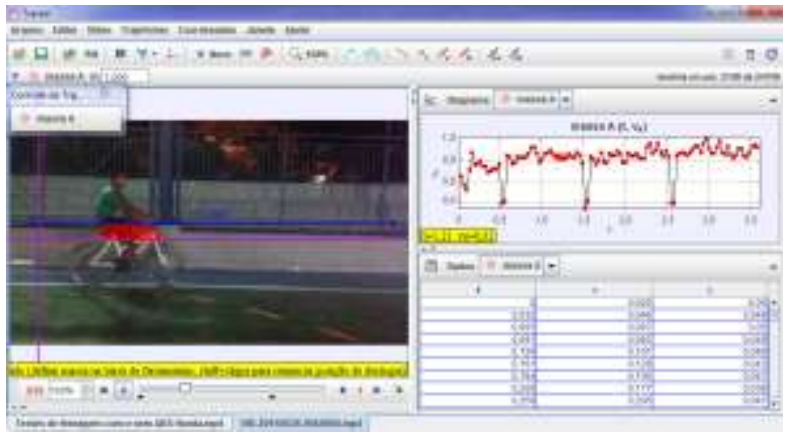

Figura 1 Análise do movimento de um ciclista e o gráfico da velocidade versus tempo, obtidos pelo software Tracker. 


\section{Resultados e discussão}

Na Figura 2, mostramos o $1^{\circ}$ teste diagnostico que foi aplicado. Ressaltamos que todas as questões eram compostas de 6 alternativas, sendo que em todas as questões a alternativa f (não mostrada na Figura 2) era não sei responder. Considere o diagrama abaixo para responder as duas
primeiras questöes. O diagrama representa um objeto
movendo-se ao longo de uma superficie horizontal. As
posiçōes indicadas no diagrama estāo separadas por
intervalos de tempo iguais. O primeiro ponto indica a
posição em que o objeto começou a se mover e último
quando ele voltou a ficar em repouso.

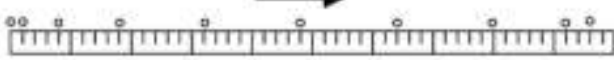

Questäo 01 - Qual dos gráficos seguintes representa melhor a velocidade do objeto em função do tempo?

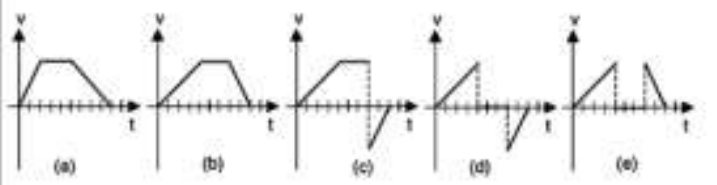

Questăo 02 - Qual dos gráficos seguintes representa melhor a aceleraçâo em funçâo do tempo?

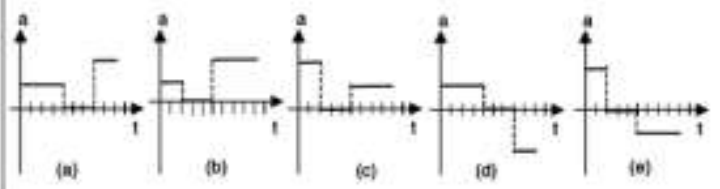

Para responder as próximas trés questoes, basele-se no gráfico da velocidade em funçäo do tempo mostrado a seguir, que representa o movimento de um objeto em uma dimensăo.

4. vim/s]

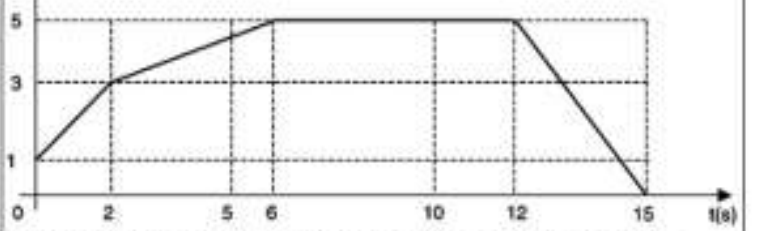

Questăo 03 - Qual è aproximadamente a aceleraçăo média do objeto entre $\mathrm{t}=0 \mathrm{~s}$ e $\mathrm{t}=6,0 \mathrm{~s}$ ?
(a) $3,0 \mathrm{~m} / \mathrm{s}^{2}$
(b) $1,5 \mathrm{~m} / \mathrm{s}^{2}$
(c) $0,83 \mathrm{~m} / \mathrm{s}^{2}$
(d) $0,67 \mathrm{~m} / \mathrm{s}^{2}$
(e) năo sei responder

Questāo 04 - Qual o espaço percorrido pelo objeto entre $\mathrm{t}=0 \mathrm{e} \mathrm{t}=6,0 \mathrm{~s}$ ?
(a) $20 \mathrm{~m}$
(b) $8,0 \mathrm{~m}$
(c) 6,0 m
(d) $1,5 \mathrm{~m}$
(e) nenhuma das anteriores

Questăo 05 - Qual è aproximadamente a velocidade média do objeto nos primeiros $6,0 \mathrm{~s}$ ?
(a) $3,3 \mathrm{~m} / \mathrm{s}$
(b) $3,0 \mathrm{~m} / \mathrm{s}$
(c) $1,8 \mathrm{~m} / \mathrm{s}$
(d) $1,3 \mathrm{~m} / \mathrm{s}$
(e) Nenhuma das anteriores.

Figura 2 - Primeiro teste diagnóstico aplicado [adaptado 9]
No Quadro 1 é apresentado o percentual de respostas assinaladas para cada um dos seis itens presentes no $1^{\circ}$ teste diagnóstico, as alternativas corretas estão em destaque. Inicialmente, duas situações saltam os olhos (i) em nenhuma questão o índice de acertos foi superior a $50 \%$ e (ii) a grande porcentagem de alunos que marcaram a alternativa " $\mathrm{F}$ - não sei responder"

Quadro 1 - Resultado geral da aplicação do $1^{\circ}$ teste diagnostico.

\begin{tabular}{|c|c|c|c|c|c|c|}
\hline Questão & $\mathrm{a}$ & $\mathrm{b}$ & $\mathrm{c}$ & $\mathrm{d}$ & $\mathrm{e}$ & $\mathrm{f}$ \\
\hline 01 & $25 \%$ & $37 \%$ & $12 \%$ & $5 \%$ & $9 \%$ & $13 \%$ \\
\hline 02 & $3 \%$ & $21 \%$ & $24 \%$ & $\mathbf{1 6 \%}$ & $15 \%$ & $21 \%$ \\
\hline 03 & $33 \%$ & $31 \%$ & $10 \%$ & $5 \%$ & $2 \%$ & $19 \%$ \\
\hline 04 & $9 \%$ & $16 \%$ & $23 \%$ & $33 \%$ & $12 \%$ & $8 \%$ \\
\hline 05 & $13 \%$ & $43 \%$ & $10 \%$ & $15 \%$ & $3 \%$ & $16 \%$ \\
\hline
\end{tabular}

$\mathrm{Na}$ questão 1, o espaçamento entre as posições da bolinha que é apresentado no enunciado está diretamente relacionado com o comportamento gráfico da velocidade. Quando analisamos a porcentagem de alunos que assinalaram as alternativas a e b, verificamos que este índice é superior a $50 \%$, o que indica que um bom número de alunos compreendeu o comportamento da velocidade no problema proposto, porém quando analisamos o percentual de acertos verificamos que uma grande parcela dos alunos que compreenderam o comportamento da velocidade não conseguiram traduzir esta observação para a linguagem gráfica.

Quando analisamos o resultado da questão 2, percebemos que houve uma distribuição praticamente homogênia no percentual de alunos que assinalou os itens de $b$ a f. $O$ alto percentual de alunos que assinalaram os itens b e c, nos fornece indicios que os alunos não conseguem relacionar de forma correta os conceitos de velocidade e aceleração e esta dificuldade é mais acentuada quando se faz necessário utilizar a linguagem gráfica. A dificuldade de compreensão da linguagem gráfica fica mais evidente quando analisamos os índices de acertos das questões 3 a 5 .

Apesar do baixo índice de acertos da questão 1 , notamos que houve uma concentração de respostas nos itens a e b, logo podemos deduzir que muitos alunos conseguiram relacionar a figura 
apresentada com o comportamento da velocidade, mas tiveram muitas dificuldades para correlacionar este comportamento com a inclinação da reta no gráfico da velocidade em função do tempo.

Esta dificuldade na utilização da linguagem gráfica fica mais acentuada quando observamos que 0 índice de acertos da questão 2 é a metade do índice de acertos da questão 1 , sendo que uma questão e continuação da outra.

Quando analisamos o resultado da questão 3, novamente, constatamos a dificuldade com a linguagem gráfica, e também temos índicios que os alunos não compreendem efetivamente a relação entre velocidade e aceleração, por exemplo: os alunos que assinalaram a alternativa b na questão 2 (21\%), cabe observar que na turma 1, nenhum aluno acertou esta questão, provavelmente calcularam a aceleração nos dois intervalos e depois tiraram a média. Com relação aos resultados das questões 4 e 5 , percebemos que quanto mais indireto for o cálculo do resultado final maior é a dificuldade apresentada.

Na Figura 3 são apresentados os índices de acertos de cada questão de cada turma avaliadas. Apesar do fato de que a turma 1 obteve um melhor resultado médio em relação a turma 2, podemos observar que o perfil de acertos das duas turmas são muito semelhante.

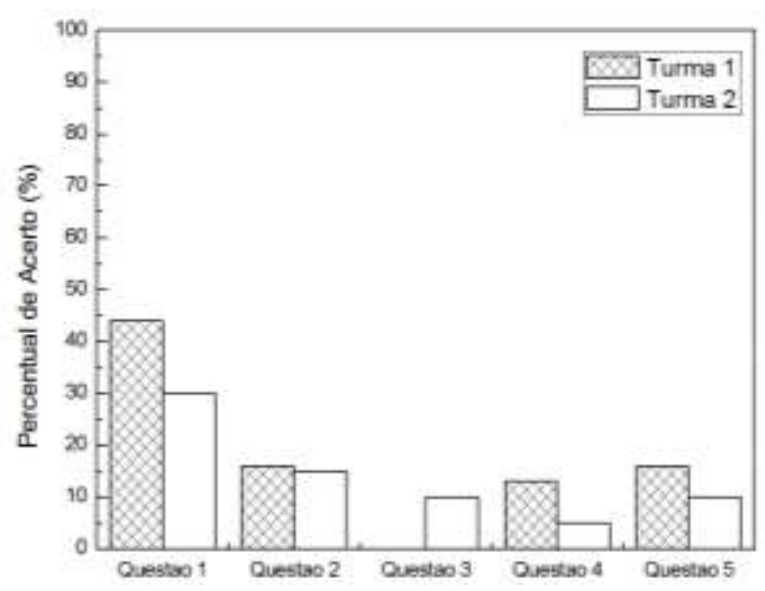

Figura 2 - Percentual de acertos que cada turma obteve no $1^{\circ}$ teste diagnóstico.

Neste ponto gostariamos de ressaltar que os resultados obtidos foram inferiores ao esperado, uma vez que estes conteúdos já haviam sido trabalhados com os alunos em sala de aula, mesmo que de forma mecanizada. Entretanto, não podemos desconsiderar que todas as questões exigem dos alunos um conhecimento básico da liguagem gráfica, neste sentido os resultados indicam que os alunos apresentam dificuldades de entender que os gráficos também são uma forma de representação de situações do cotidiano.

Após a aplicação do $1^{\circ}$ teste diagnostico, foi realizada a aula, onde os vídeos foram analisados com o software Tracker. O principal objetivo, nesta aula era enfatizar que os gráficos são uma linguagem eficiente na descrição de situações cotidianas. É importante ressaltar que os alunos não tiveram acesso ao resultado final e ao gabarito do $1^{\circ}$ teste diagnóstico.

No momento subsequente, aplicamos o $2^{\circ}$ teste diagnóstico e os resultados estão sumarizados no Quadro 2, as alternativas corretas estão em destaque. Vale lembrar que a ordem das questões no $2^{\circ}$ teste diagnóstico não é a mesma do $1^{\circ}$ teste.

Quadro 2 - Resultado geral da aplicação do $2^{\circ}$ teste diagnóstico.
\begin{tabular}{|c|c|c|c|c|c|c|}
\hline Questão & a & b & c & d & e & $f$ \\
\hline 01 & $0 \%$ & $0 \%$ & $97 \%$ & $2 \%$ & $1 \%$ & $0 \%$ \\
\hline 02 & $8 \%$ & $15 \%$ & $71 \%$ & $4 \%$ & $2 \%$ & $0 \%$ \\
\hline 03 & $15 \%$ & $10 \%$ & $58 \%$ & $10 \%$ & $5 \%$ & $2 \%$ \\
\hline 04 & $66 \%$ & $8 \%$ & $12 \%$ & $4 \%$ & $6 \%$ & $4 \%$ \\
\hline 05 & $64 \%$ & $13 \%$ & $5 \%$ & $6 \%$ & $5 \%$ & $7 \%$ \\
\hline 06 & $12 \%$ & $52 \%$ & $15 \%$ & $11 \%$ & $6 \%$ & $4 \%$ \\
\hline
\end{tabular}

Neste ponto, ressaltamos o significativo aumento no percentual médio de acertos e o decrescimo da porcentagem de alunos que assinalaram as alternativas $\mathrm{F}$ (não sei responder). $\mathrm{O}$ resultado da questão 1 (97 \% de acertos), mostra que os alunos sabem qual a grandeza pode ser calculada a partir do gráfico, uma vez que esta questão foi inserida no $2^{\circ}$ teste diagnostico porque durante a aula os alunos apresentaram dúvidas com relação as possíveis informações que podem ser retiradas dos gráficos.

O alto índice de acertos da questão 2, cerca de $71 \%$, foi o resultado que mais chamou atenção, pois esta questão corresponde a questão 3 do $1^{\circ}$ teste, que teve um índice médio de acertos de 5\%. Mesmo diante deste bom resultado percebemos que quando os cálculos ficam mais indiretos, o percentual de acertos diminui. 
$\mathrm{Na}$ Figura 4, temos um comparativo entre os índices de acertos de cada turma no $2^{\circ}$ teste diagnóstico. Podemos notar que em ambas as turmas ocorreu uma melhora no desempenho dos alunos avaliados, sendo que esta melhora foi mais acentuada na turma 2. Diante deste cenário é possível afirmar que existem índicios de que a utilização do software Tracker contribuiu para diminuir as dúvidas que os alunos possuem nos conteúdos abordados.

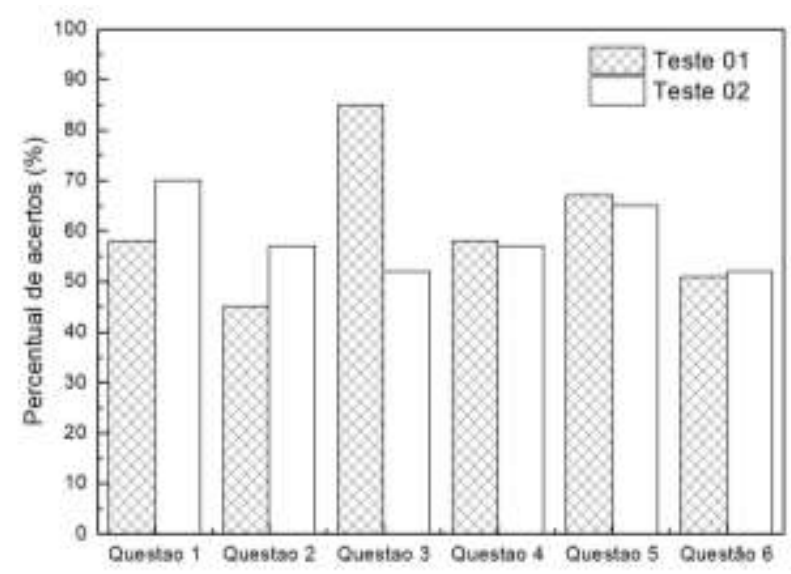

Figura 3 - Percentual de acertos que cada turma obteve no $2^{\circ}$ teste diagnóstico.

\section{Conclusões}

Neste trabalho tivemos como objetivo principal relatar a utilização do software Tracker como um recurso auxiliar no ensino do movimento e, fornecer indícios sobre o efeito da utilização deste tipo de ferramenta no entendimento e motivação dos alunos. Para isto, trabalhamos situações lúdicas, como movimentos presentes em situações cotidianas dos alunos. Os resultados obtidos através dos testes diagnósticos mostraram uma melhoria no aprendizado dos alunos, sendo que com apenas uma aula de 50 minutos onde aplicamos do programa, o percentual médio de acertos nos testes diagnósticos foi de $14 \%$ para $78 \%$. Além disso, os alunos demostraram um melhor entendimento dos conceitos físicos.

O programa mostrou-se bastante útil e prático na utilização em sala de aula, além de ser um programa gratuito e de fácil manuseio em sua aplicação, mostrou-se também um excelente atrativo para os alunos facilitando o processo de aprendizagem.

Acreditamos que nossos resultados indicam que o uso do Tracker contribuiu para melhorar o interesse, a participação ativa dos alunos, a consciência e o fato de que física não se resume a um conjunto de expressões matemáticas, o entendimento de que eventos físicos podem ser descritos e representados através de gráficos e a constatação de que situações do cotidiano podem ser estudadas e analisadas de forma a serem descritas por equações matemáticas. Entretanto ressaltamos que esta é uma ferramenta auxiliar e que não é possível ensinar todos os conteúdos desta forma.

\section{Referências}

[1] BARBOSA, M.A.; SILVA, W.C., Scientia Plena, v.4, n.12, 2008.

[2] BONADIMAN, H.; NONENMACHER, S. E. B., Cad. Bras. Ens. Fís., v. 24, n. 2: p. 194-223, ago. 2007.

[3] CLEMES, G. et al. Vídeo-aula como estratégia de ensino de Física. In: 1ำ Simpósio de Integração Científica e Tecnológica do Sul Catarinense - SICTSul, 2012. Anais eletrônicos. Disponível em file:/// C:/Users/Marcio/Downloads/597-2357-2-PB\%20(3).pdf. Acesso em: 15 de março de 2015.

[4] TRACKER - Video analysis and modeling tool. Disponível em < http://physlets.org/tracker/>. Acesso em: 03 de março de 2015.

[5] CALLONI, G.J. A física dos movimentos analisada a partir de vídeos do cotidiano do aluno: Uma proposta para oitava série. Dissertação (Mestrado em Ensino de Física), Instituto de Física, Universidade Federal do Rio Grande do Sul, Rio Grande do Sul, 2010.

[6] BEICHNER, R. Journal of research in science teaching, v.27, $n^{\circ} 8$, p. $803-815,1990$.

[7] BEICHNER, R., American Journal of physics, v. 64, p.1272, 1996.

[8] BARBETA, V.B., YAMAMOTO, I., Revista Brasileira de Ensino de Física, v. 24, nº. 2, p. 158 - 167, 2002.

[9] HESTENES, D., WELLS, M., The Physics Teacher v. 30 , p. 159 - 166, março 1992. 